ber of proton bunches has been increased to 170 and the bunch current increased to 300 $\mathrm{mA}$. A sudden, unpredictable reduction in electron lifetime is attributed to the capture of micro-particles produced in the sputter-ion pumps and accelerated in the beam. The threshold for this effect has been increased by a factor of 5 by replacing a short length of beam pipe with integrated pumps. The big success story has been the production of longitudinally polarized beams following the installation of a spin rotator. With the rotator switched off, careful optimization produced $60 \%$ transverse polarization. The spin rotator was then switched on and almost miraculously, without any further adjustments, the longitudinal polarization rose to nearly $60 \%$ in just over one hour. HERA is now essentially running routinely with longitudinally polarized electrons.

Major new facilities are under construction, notably the Continuous Electron Beam Accelerator Facility (CEBAF) consisting of two $0.4 \mathrm{GeV}$ linacs through which the beam passes 5 times giving an extraction energy of $4 \mathrm{GeV}$. Andrew Hutton reported that the linac had reached $110 \mathrm{~mA}$ (design $200 \mathrm{~mA}$ ), and that a cryomodule of superconducting cavities had achieved a gradient of $8 \mathrm{MV} / \mathrm{m}$ with a beam (design: $5 \mathrm{MV} / \mathrm{m}$ ). Dave Burke (Stanford Linear Accelerator Center) reported that the Final Focus Test Beam (FFTB) collaboration's very impressive test facility had met its design objective by producing beam spot sizes having the same demagnification as those assumed in designs for future linear colliders. Hospital-based radiation therapy units remain a dominant theme and Marco Silari (Milan) summarised an ambitious 1000 patients per year, hadron-therapy complex for Italy comprising a $\mathrm{H}$ synchrotron $(60-250$ $\mathrm{MeV} / 10-20 \mathrm{nA}$ proton beams). Two extraction channels would operate independently, with a possible upgrade to allow acceleration of light ions up to $400 \mathrm{MeV} / \mathrm{u}$.

\section{Accelerator Physics and Components}

In a comprehensive report on the status of superconducting cavities, Bernhard Bonin (Saclay) pointed out that accelerating gradients in superconducting structures have been increased significantly in the last few years owing to improved cleaning techniques, high peak power RF processing, higher purity niobium, and improved heat treatments. High gradients have been achieved in several laboratories (notably for $3 \mathrm{Ghz}$, $1.8 \mathrm{~K}, 9$-cell cavities made at Cornell and Wuppertal). Many are confident that these cavities will reach surface fields as high as 30 $\mathrm{MV} / \mathrm{m}$ reliably (corresponding to a usable gradient of $15 \mathrm{MV} / \mathrm{m}$ ). Nonetheless, while RF superconductivity has come of age it is still a long way from its ultimate possibilities.

In a very interesting presentation on a $\mathrm{mm}$ wave linac and wiggler structures, Heino Henke (TU, Berlin) presented a new technology for fabricating accelerator components and wigglers based on silicon technology and high precision micro-mechanics (his pilot design was for a $50 \mathrm{MeV}$ millimetre RF wavelength electron linear accelerator complex for producing coherent, tuneable synchrotron radiation - see cover). Solutions are apparently limited more by our imagination than by cost!

Comparisons of calculated and measured dynamic apertures in HERA were discussed by Frank Zimmerman (SLAC). This is an

Martin Wilson, the Chairman of Oxford Instruments, speaking during the "Technology Transfer between Accelerator Laboratories and Industry" session which was organized and chaired by Neil Marks (Daresbury). It ended with an animated round-table discussion, led by Sergio Tazzari (INFN, Frascati), on the various needs for the future industrial development of accelerator technology.
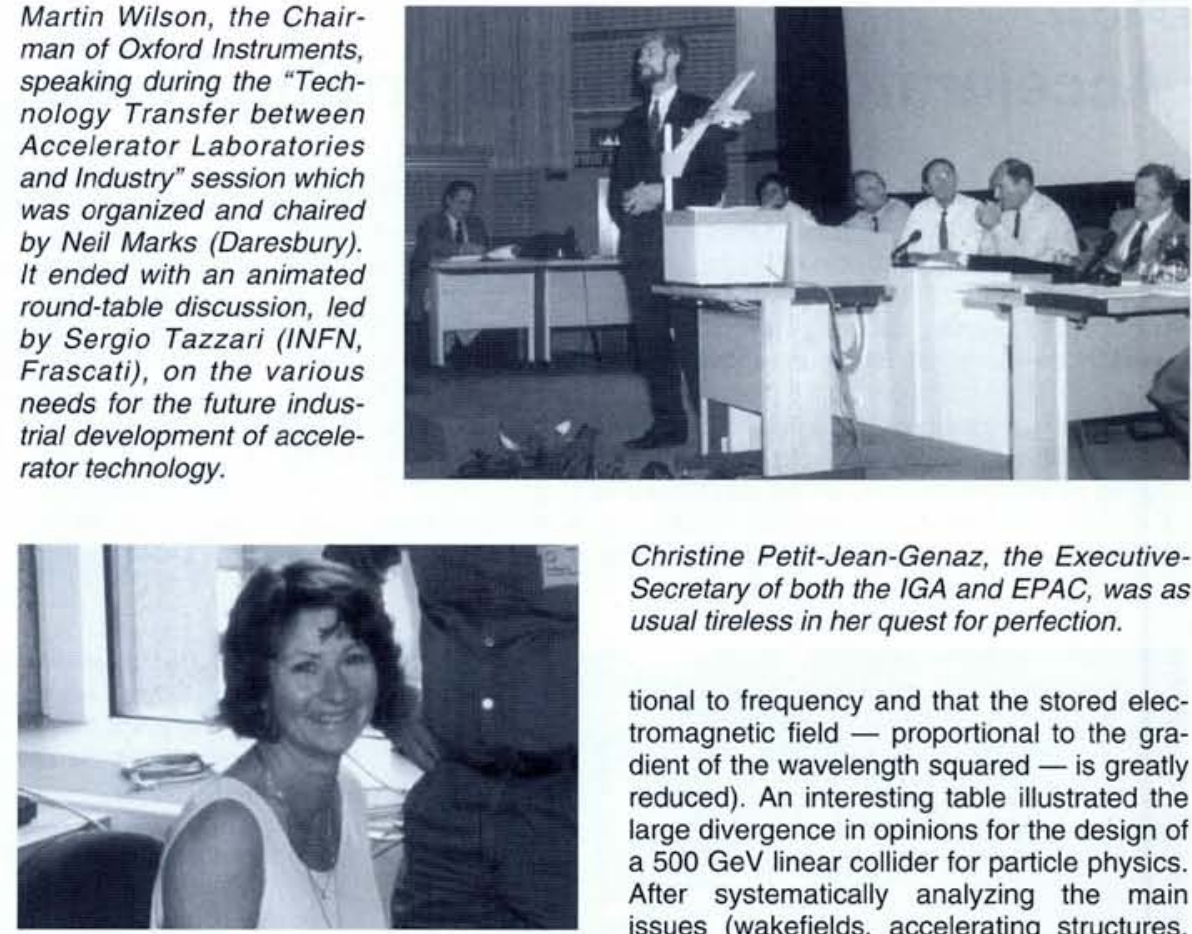

Christine Petit-Jean-Genaz, the ExecutiveSecretary of both the IGA and EPAC, was as usual tireless in her quest for perfection.

tional to frequency and that the stored electromagnetic field - proportional to the gradient of the wavelength squared - is greatly reduced). An interesting table illustrated the large divergence in opinions for the design of a $500 \mathrm{GeV}$ linear collider for particle physics. After systematically analyzing the main issues (wakefields, accelerating structures, emittance control, RF power, final focus) with respect to the frequency, he concluded that a few more years of research are needed, along with exploitation of the various existing and proposed test facilities, in order to make a well-founded choice between the various proposals.

Accelerator-based mass spectrometry is an example of the diversity of modern accelerator physics and technology and the extent to which they impinge upon science. M. Suter (ETH, Zurich) described in detail the measurement of small concentrations of tracer isotopes which is the basis of dating using long-lived radioisotopes (for example, the Tyrolean iceman's age has been determined to be 4500 years). With presentations up to the last day on topics ranging from $\Phi$-factories, accelerator research, medical applications, and high-energy beams in the universe, including cosmic rays, it is no wonder that most participants remained until the final talk before heading out into the hot, untypically British weather which we had enjoyed throughout the conference.

S. Myers, CERN allow a higher accelerating gradient (since the onset gradient of dark current is propor-

The Editor reviews the special session at EPAC'94 entitled "High Intensity Issues".

\title{
High Beam Intensity Comes to the Fore
}

\section{Further Progress Needs New Machines}

"Interesting applications that force innovation and development" was how Stan Schriber (Los Alamos National Laboratory) concluded his survey at EPAC'94 of proposed high-intensity accelerators and their applications. One is considering here accelerators with an average beam power of at least about $1 \mathrm{MW}$, typically $10-300 \mathrm{~mA}$ at 0.5-2.0 GeV as compared with existing stateof-the-art machines offering $45 \mathrm{~mA}$ at 35 MeV (Fusion Material Test Facility, Los Alamos,: commissioned in 1984) and $1 \mathrm{~mA}$ at $800 \mathrm{MeV}$ (LAMPF, Los Alamos; commissioned in 1972). Using such devices, free- electron laser and neutral particle beam defense systems "got somewhere" before the US Space Defense Initiative discarded the directed energy approach. The technology that was developed is now being used elsewhere, and with the declassification last year of the US's defense-related work understanding will be extended.

Generally speaking, all reasonable applications of high-intensity accelerators need well thought out, robust, industrial-style, costeffective, and safe designs with a high operating efficiency, a high power conversion efficiency and hands-on maintenance while ensuring precision control at high beam cur- 


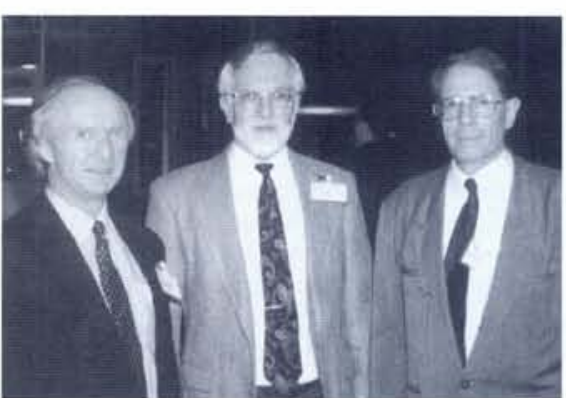

Horst Klein, who organized the session on high intensity issues, with Stan Schriber (centre) and M. Craddock from TRIUMF (on the left), the session chair.

rents. Given these formidable requirements one might ask where to begin. Concentrating on the beam physics, George Lawrence of the beam emittance along a high-intensity linac (since aperture and cost must be kept to tolerable levels) and beam losses (to ensure little activation of components). Work during the last decade will provide a viable starting framework for controlling emittance growth, which is mainly determined by mismatches between the different accelerator sections in the longitudinal and transverse phase tracking are now being used to simulate beam losses, and in particular halo formation (using additional analytical modelling). Until understanding matures, the design criteria to achieve low beam losses will be based on operating experience, where it seems that one can be confident that hands-on maintenance should be possible for the various high-intensity linacs being proposed for several major applications (see table).

\section{Bridging the Gap}

Ingo Hofmann (GSI, Darmstadt) showed that in the case of high intensity, spacecharge dominated beams in storage rings (as might be used in heavy-ion driven inertial confinement fusion) where one tries to push longitudinal and transverse space charge to the limit, it is possible to bridge the gap (LANL) argued that the key issues are growth spaces. Computer codes for multiparticle

\section{EPS-IGA Accelerator Prize}

The EPS-IGA Accelerator Prize is awarded without restriction as to nationality to a physicist or engineer, in the early part of his or her career, who has made a recent, original, and significant contribution to the field of particle accelerators. Grahame Rees from RAL (on the right), who chaired the Prize Committee, is shown here presenting the first prize jointly to Igor Syrachev (Institute of Nuclear

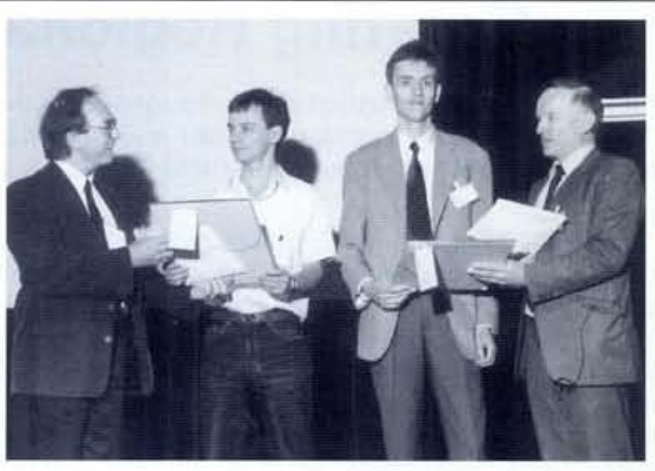
Physics, Protvino; third from left) for work on an open cavity RF pulse compression system, and to Hákan Danared (Manne Siegbahn Laboratory, Stockholm; second from left) for improvements to electron cooling. Syrachev, in a very talented piece of research, designed a new type of cavity that has advantages for pulsed beams while Danared revisited an approach that had been tried before and managed to get it to work. Michel Olivier, the EPAC Organizing Committee chair, is on the left.

Bridging experiment and theory: excellent agreement is found between the power spectrum of the longitudinal noise in a simulated particle beam (a, upper), calculated by taking a Fourier transform of the fluctuations with time of the line density, and the measured noise spectrum (b, lower) for $3 \times 10^{8} \mathrm{Ne}^{10+}$ particles in a cooled bunch at $250 \mathrm{MeV} / \mathrm{u}$. The noise amplitude (a) and the rms noise (b) are plotted as functions of the frequency. Moreover, there is good agreement between the parameters of the various oscillations calculated from the simulated noise and from analytical formulae. The agreement is not so good for the transverse noise, indicating that the analytical models are inadequate (at least for the types of bunches studied so far). Figures courtesy of I. Hofmann, GSI, Darmstadt.

between simplified theoretical models and experiments on real beams by comparing measured noise spectra with "simulation noise" obtained from computer simulations of interacting particles. The various beam oscillations are clearly revealed (see figure) and there is agreement between oscillation parameters estimated using analytical formulae and calculated for the simulation noise.

\section{High-intensity accelerator-driven transmutation technologies}

\section{Heavy-ion drivers for inertial confinement fusion}

- heavy-ion beam implodes a fuel pellet

Design study to be proposed to the European Commission; linac + storage ring.

Pulsed spallation neutron sources for research

- protons produce neutrons in a heavy metal target by a spallation process European Spallation Source (ESS) design study in progress; $4.2 \mathrm{~mA} / 1.2 \mathrm{GeV}$ proton linac + accumulator ring.

Neutron source for radiation damage studies of fusion reactor vessels - deuteron accelerator producing neutrons by stripping in a Li target

IFMIT (International Fusion Materials Irradiation Test) facility design study close to approval by EURATOM: 2 or 4 approximately $250 \mathrm{~mA} / 30-40 \mathrm{MeV}$ variable energy, continuous-wave linacs in parallel.

Materials production $\left(\mathrm{Tr}^{+}, \mathrm{P}, \mathrm{U}\right)$ and destruction ( $\mathrm{P}$, nuclear waste $\left.{ }^{*}\right)$

- "to convert long-lived actinides and fission products using either a heavy metal spallation source to generate fast neutrons that are absorbed in the waste or protons producing high fluxes of thermal neutrons in a target blanket

Los Alamos proposal to the US Dept. of Energy: $100 \mathrm{~mA} / 7 \mathrm{MeV} \mathrm{CW}$ proton linac front end technology demonstrator (LAMPF upgrade) for a $200 \mathrm{~mA} / 1000 \mathrm{MeV}{ }^{+} \mathrm{ATP} /{ }^{\star} \mathrm{ATW}$ complex to fully demonstrate the feasibility of "funnelling" two beams into one.

\section{Nuclear energy production}

- energy amplification using energy cascades induced by accelerated protons Preliminary experiments at CERN to test feasibility planned.
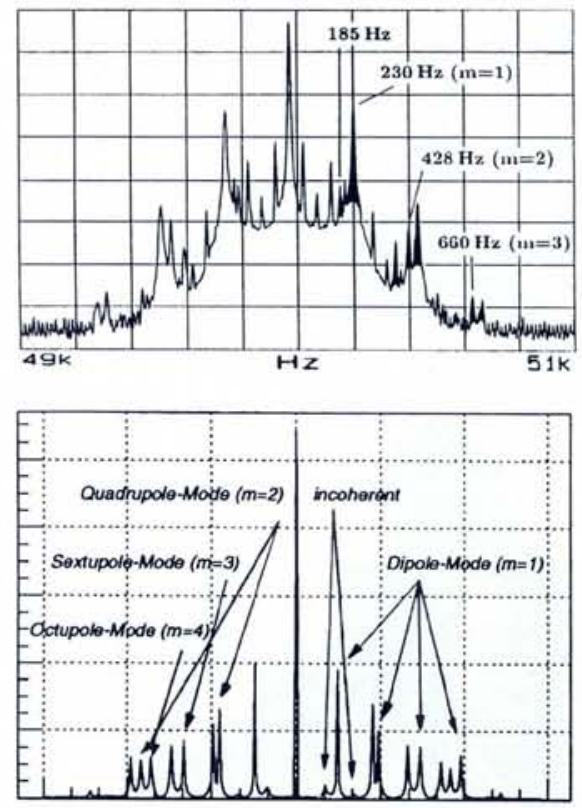

frequency

A similar approach may one day help in making sense of simulations of the all-important beam halo, notably its formation, dynamics, amplitude, and dependence on space charge, because to understand the halo one needs to understand the oscillations. Advanced methods for the real-time observation of high-phase space beams (see cover illustration) will also help bridge the gap, as demonstarted by Ingo Hofmann's first streak camera observations of cooled, heavy-ion beams which offer excellent space and time resolution simultaneously.

But understanding will only be consolidated by going to the next stage by building and operating a next-generation, high energy/high current accelerator, either as a firststage linac demonstrator for a larger facility (the Los Alamos ATP/ATW proposal) or a pulsed neutron source facility (the ESS proposal). The entire accelerator complex will come under close scrutiny, including the high-intensity source, the low-energy part, high output current storage rings (if they are used), diagnostics, and control systems, each of which was reviewed in some detail by speakers. 